Editor's Note: These short, critical reviews of recent papers in the Journal, written exclusively by graduate students or postdoctoral fellows, are intended to summarize the important findings of the paper and provide additional insight and commentary. For more information on the format and purpose of the Journal Club, please see http://www.jneurosci.org/misc/ifa_features.shtml.

\title{
Spindle-Derived NT3 in Sensorimotor Connections: Principal Role at Later Stages
}

\author{
Svetlana Gorokhova, Stéphane Gaillard, and Eduardo Gascon \\ Developmental Biology Institute of Marseille Luminy, 13288 Marseille Cedex 9, France \\ Review of Shneider et al.
}

The circuit between muscle spindles, a subtype of proprioceptors (Ia afferents), and motor neurons is established during late embryonic development and is essential for proper coordination and movement. Muscle spindles, the specialized sensory structures that respond to muscle stretch, are induced by sensory axon innervation around embryonic day 15. As connections between proprioceptive neurons and muscle spindles are being formed in the periphery, central afferents of these neurons are completing the final axonal extension toward the ventral spinal cord, where they will form connections with motor neurons.

Some evidence suggests that muscle spindles produce signals that influence the establishment of functional central connections (for review, see Chen et al., 2003). One spindle-derived signal that might be responsible for development of sensorimotor connections is neurotrophin-3 (NT3). NT3 induces sensory neuron expression of ER81, a transcription factor that controls the extension of the central sensory projections toward the ventral spinal cord. Indeed, in NT3/Bax double-knockout mice, in which the neuronal cell death due to NT3 absence is prevented

Received June 11, 2009; revised July 17, 2009; accepted July 21, 2009.

We thank Françoise Helmbacher and Aziz Mogrich for comments on this manuscript.

Correspondence should be addressed to Svetlana Gorokhova, Developmental Biology Institute of Marseille Luminy, Case 907, Parc Scientifique de Luminy, 13288 Marseille Cedex 9, France. E-mail: gorokhova@ibdml.univ-mrs.fr. D0I:10.1523/JNEUROSC1.2741-09.2009

Copyright $\odot 2009$ Society for Neuroscience $\quad$ 0270-6474/09/2910181-03\$15.00/0 by deleting the proapoptotic protein Bax, the afferent axons do not extend beyond the intermediate spinal cord, a phenotype similar to that of mice lacking ER81 (Arber et al., 2000; Patel et al., 2003). Muscle spindles are either abnormal or absent in both of these mutants.

In contrast to these results, central connections appear to project normally to ventral spinal cord in other mouse models, despite severely compromised development of muscle spindles. For example, no gross defects in central projections were observed in Neuregulin 1 knock-out mice, in which muscle spindles were not induced (Hippenmeyer et al., 2002). Similarly, central projections in mice lacking the Neuregulin 1 receptor ErbB2 in muscles appear to project normally to ventral cord, even though muscle spindles in these mice are immature and greatly reduced in number (Leu et al., 2003). On the other hand, the presence of grossly normal Ia afferent projection patterns in the spinal cord does not necessarily mean that sensorimotor connections are functional. Indeed, monosynaptic responses are greatly reduced postnatally in Egr3-null mice, in which central projections extend to ventral spinal cord, but spindles lack NT3 and degenerate after birth (Chen et al., 2002). Peripheral injection of NT3 restores the monosynaptic response in these mice, suggesting that spindle-derived NT3 influences the strength of sensorimotor connections (Chen et al., 2002).

Thus, recently described mouse mutants with absent or defective spindles can be divided into two major groups: those in which central projections fail to extend past the intermediate spinal cord and those with grossly normal central projection morphology, but possible postnatal reduction in sensorimotor synaptic strength. Despite recent advances, several important questions remain. In the mutants with apparently normal ventral projections of Ia afferents, do sensorimotor connections form with correct specificity? Do muscle spindles play a role in establishment or maintenance of these connections? What is the role of NT3 in this process? Shneider et al. (2009) address these questions in their recent article in The Journal of Neuroscience.

Shneider et al. (2009) generated mice with conditionally deleted ErbB2 in intrafusal muscle fibers (ErbB2 $\Delta$ mice). Like in other mice with muscle-specific ErbB2 mutation, spindles in ErbB2 $\Delta$ mice were highly immature and greatly reduced in numbers, while central Ia afferent projections had a grossly normal appearance [Shneider et al. (2009), their Fig. 1, Fig. 2A-F]. The authors determined that direct contacts formed between proprioceptive afferents and motor neurons in ErbB2 $\Delta$ animals [Shneider et al. (2009), their Fig. 2D-L]. Quantification of these contacts in adult animals showed a $30 \%$ reduction in contact density in mutant animals [Shneider et al. (2009), their Fig. 3], suggesting that despite the absence of normal spindles in the periphery, the majority of contacts between sensory afferents and motor neurons were still present in adulthood. 
Shneider et al. (2009) tested the specificity of monosynaptic connections in ErbB2 $\Delta$ mice by measuring the latency of activation of functionally antagonistic LG and TA motor neurons upon stimulation of either homonymous or antagonist muscle nerves. These latencies were similar between ErbB2 $\Delta$ and wild-type mice, suggesting that connections between afferents and motor neurons were specific in the mutant animals [Shneider et al. (2009), their Fig. 8]. The authors further confirmed the specificity of sensorimotor connections by labeling afferents from LG and TA muscles [(Shneider et al. (2009), their Fig. 9].

To assess the functionality of sensorymotor connections, the authors measured the amplitude of the monosynaptic response in ErbB $2 \Delta$ and control animals by recording from motor neurons as well as ventral roots, while verifying motor pool identity by both retrograde tracer labeling and intracellular dye filling. The results of this experiment showed that the amplitude of monosynaptic potentials in ErbB $2 \Delta$ mice was reduced by $80 \%$ compared with wild-type animals [Shneider et al. (2009), their Fig. 6C,D]. Thus, even though afferent axons extended to ventral spinal cord and made specific contacts with motor neurons, the motor neuron response to sensory axon stimulation was diminished.

Could defects in ErbB2 $\Delta$ spindle development be responsible for the attenuation of monosynaptic responses? Since mutant spindles in ErbB2 $\Delta$ animals failed to express NT3 [Shneider et al. (2009), their Fig. $1 C, F]$, it is possible that lack of this neurotrophin caused the observed changes in central connections. To test this hypothesis, Shneider et al. (2009) conditionally eliminated spindle-derived NT3 in vivo, specifically in muscle spindles and not in surrounding muscle fibers (NT3 $\Delta$ mice). Surprisingly, despite absence of spindle NT3, the amplitude of the afferent-evoked monosynaptic response recorded in the ventral roots of NT3 $\Delta$ mice during the first postnatal week (measured at postnatal day 5) did not differ from that of wild-type controls. However, when tested at postnatal day 14 , the amplitude of the monosynaptic ventral root potentials in the mutant mice was reduced by $>80 \%$ [Shneider et al. (2009), their Fig. $7 G-J]$. Similar results were also obtained when NT3 was deleted from all muscle fibers. Although connectivity and specificity was not tested directly in NT3 $\Delta$ animals, normal monosynaptic connections during the first postnatal week indi-

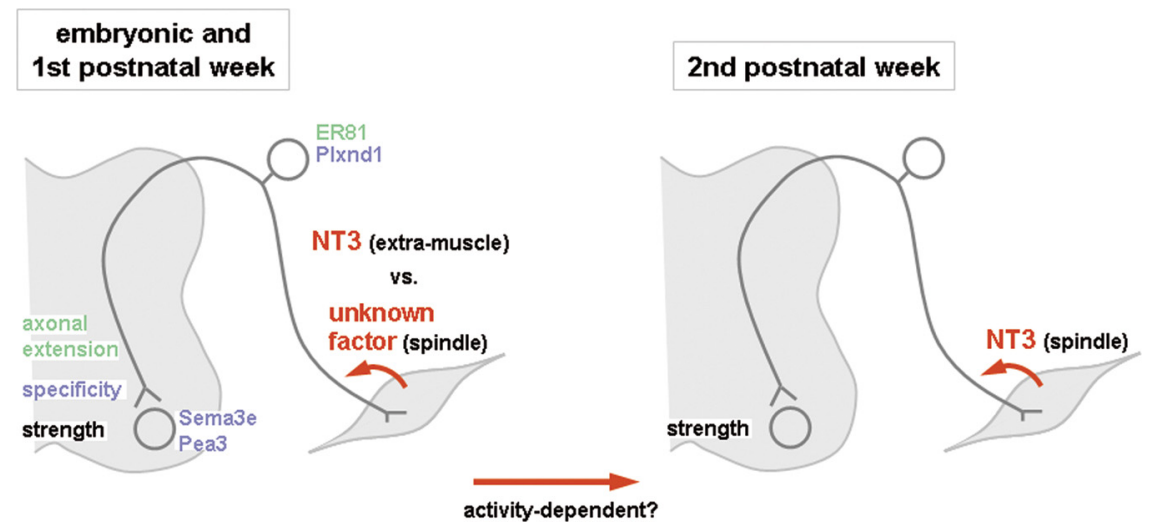

Figure 1. Spindle-derived NT3 is important for functional sensorimotor connections after the second postnatal week. Before that, extramuscle NT3 and/or unknown spindle-derived factors are likely to play a role in the establishment and functional development of connections between sensory afferents and motor neurons. This change in dependence toward spindle-derived NT3 could be a consequence of increased muscle activity during the second postnatal week. Molecules that play a role in axonal extension and in defining the specificity of monosynaptic connections during embryonic development are shown in green and blue, respectively.

cate that axonal extension and specificity of sensory-motor connections are determined independently of muscle-derived NT3. Moreover, the strength of monosynaptic connections depends on spindlederived NT3 only after the second postnatal week.

From the results of this study, Shneider et al. (2009) draw two main conclusions: first, spindle-derived factors influence the strength of central sensorimotor connections but not their specificity; and second, spindle-derived NT3 plays a role in determining the strength of these connections only after the second postnatal week. While the first conclusion is in line with previous reports, such as the presence of abnormal spindles but normal monosynaptic connections at postnatal day 0 in mice lacking Egr3, the fact that spindlederived NT3 is dispensable during development and first postnatal week is much more surprising. NT3 has long been thought to be crucial for establishing central connections during development (Arber et al., 2000; Patel et al., 2003), as well as influencing the function of these connections postnatally (Chen et al., 2002). However, the source of NT3 remained a point for debate. The study by Shneider et al. (2009) clearly excludes both spindle-derived and, more generally, muscle-derived NT3 from playing a role during embryonic and early postnatal stages. A summary of spindle and NT3 roles during embryonic and postnatal development is shown in Figure 1.

If spindles and muscles are not important for specificity of the central connections, what is the source of the peripherally derived signal? One possibility is that development of central projections is regulated by NT3 produced by mesenchymal cells. Indeed, it has previously been shown that NT3 is strongly expressed around growing sensory axons during development (Fariñas et al., 1996). Conditionally deleting NT3 from mesenchymal cells could help to answer this question. Another possibility is that the semaphorin Sema3e, which is expressed in motor neurons, and its high-affinity receptor plexin D1, present in sensory neurons, specify the wiring of the monosynaptic connections, as suggested by Pecho-Vrieseling et al. (2009). Whether plexin D1 expression in proprioceptive neurons is regulated by extramuscle NT3 or other peripherally derived signals remains to be determined.

An alternative explanation for the fact that spindle-derived NT3 is dispensable during development and first postnatal week is that another spindle-derived factor regulates the establishment and functional development of central connections during this time. One way to distinguish between these two possibilities is to conditionally express the diphtheria toxin receptor in muscle spindles, and then to specifically ablate spindles at different points of embryonic or postnatal development by injecting diphtheria toxin. In this case, the influence of any spindlederived factor would be eliminated, while leaving the extraspindle source of NT3 intact.

Another question that emerges from the results of Shneider et al. (2009) is why do the central connections become dependent on spindle-derived NT3 only during the second postnatal week? One probable explanation is that this switch is 
activity dependent. Even though the correct pattern of connections is thought to be established independently of electrical activity during embryonic development, the strength of connections postnatally is likely to depend on activity of the circuit. It is possible that the first postnatal week is an extension of the embryonic period when the general circuit is formed, while the refinement of the connections, such as strengthening of some central synapses and elimination of others, happens when animals start to move and is dependent on spindle-derived NT3. Pharmacological suppression of muscle activity at different stages of postnatal development could help test this hypothesis.

Although many questions remain, the study by Shneider et al. (2009) highlights the limited role of spindles in the establishment of sensorimotor connections and provides key evidence that spindlederived NT3 is important starting from the second postnatal week.

\section{References}

Arber S, Ladle DR, Lin JH, Frank E, Jessell TM (2000) ETS gene Er81 controls the formation of functional connections between group Ia sensory afferents and motor neurons. Cell 101:485-498.

Chen HH, Tourtellotte WG, Frank E (2002) Muscle spindle-derived neurotrophin 3 regulates synaptic connectivity between muscle sensory and motor neurons. J Neurosci 22:3512-3519.

Chen HH, Hippenmeyer S, Arber S, Frank E (2003) Development of the monosynaptic stretch reflex circuit. Curr Opin Neurobiol 13:96-102.

Fariñas I, Yoshida CK, Backus C, Reichardt LF (1996) Lack of neurotrophin-3 results in death of spinal sensory neurons and premature differentiation of their precursors. Neuron 17:1065-1078.
Hippenmeyer S, Shneider NA, Birchmeier C, Burden SJ, Jessell TM, Arber S (2002) A role for neuregulin1 signaling in muscle spindle differentiation. Neuron 36:1035-1049.

Leu M, Bellmunt E, Schwander M, Fariñas I, Brenner HR, Müller U (2003) Erbb2 regulates neuromuscular synapse formation and is essential for muscle spindle development. Development 130:2291-2301.

Patel TD, Kramer I, Kucera J, Niederkofler V, Jessell TM, Arber S, Snider WD (2003) Peripheral NT3 signaling is required for ETS protein expression and central patterning of proprioceptive sensory afferents. Neuron 38:403-416.

Pecho-Vrieseling E, Sigrist M, Yoshida Y, Jessell TM, Arber S (2009) Specificity of sensorymotor connections encoded by Sema3ePlxnd1 recognition. Nature 459:842-846.

Shneider NA, Mentis GZ, Schustak J, O'Donovan MJ (2009) Functionally reduced sensorimotor connections form with normal specificity despite abnormal muscle spindle development: the role of spindle-derived neurotrophin 3. J Neurosci 29:4719-4735. 